\title{
Fuzzy Group Decision Making for Multiobjective Problems: Tradeoff between Consensus and Robustness
}

\author{
Jian Xiong, ${ }^{1}$ Xu Tan, ${ }^{2}$ Ke-wei Yang, ${ }^{1}$ and Ying-wu Chen ${ }^{1}$ \\ ${ }^{1}$ Department of Management, College of Information System and Management, National University of Defense Technology, \\ Changsha, Hunan 410073, China \\ ${ }^{2}$ School of Software, Shenzhen Institute of Information Technology, Shenzhen 518172, China
}

Correspondence should be addressed to Jian Xiong; xiongjian1984@hotmail.com

Received 3 April 2013; Revised 24 June 2013; Accepted 12 July 2013

Academic Editor: Jianming Zhan

Copyright (C) 2013 Jian Xiong et al. This is an open access article distributed under the Creative Commons Attribution License, which permits unrestricted use, distribution, and reproduction in any medium, provided the original work is properly cited.

\begin{abstract}
Many decision making problems involve multiple decision makers and conflicting objectives. This paper refers to this kind of problems as group decision making for multiobjective problems (GDM-MOP). The task of GDM-MOP is to select final solution(s) from a set of nondominated solutions according to the decision makers' preferences. However, it is common that the preference could be imprecise. We study the GDM-MOP where preferences are expressed by fuzzy reference points, called as fuzzy GDMMOP (FGDM-MOP). This paper provides a decision support model to simultaneously consider two measures for FGDMMOP: consensus measure and robustness measure. The former is used to reflect the acceptable degree of a solution by the decision making group, while the latter indicates a solution's ability to cope with any change on preferences. A multiobjective evolutionary approach is presented to solve the problem. Finally, a modified benchmark function is studied to illustrate the proposed approach.
\end{abstract}

\section{Introduction}

Many real-world decision problems involve multiple decision makers (DMs). For instance, decisions on a corporation's investment policy may need discussion and negotiation amongst the members of directorate, or alternative selection of a complex project needs the consultation of experts from different fields. Such decision problems are referred to as group decision making (GDM) problems. Many studies suggest that GDM is appropriate to be applied for arriving at a judgment based on the input and feedback of multiple individuals [1-3]. In the process of GDM, for each alternative or comparison of a pair of alternations, DMs need to provide their opinions or preferences on one or more evaluating criteria. Then the individual opinions are aggregated to form the collective one to select the final solution. However, in practice, it is very common that decisions need to be made with simultaneous consideration of multiple conflicting objectives; that is, DMs need to trade off between different objectives. Such problems are called as multiobjective problems (MOPs), and the term multiobjective optimization (MOO) refers to solving MOPs
[4]. The results of MOPs are a set of mathematically equally good solutions, referred to as non-dominated set, among which any decision on the solutions cannot be made until the preference of decision maker(s) is provided. Usually, it is worthwhile to assist decision makers in refining obtained solutions. Thus, MOO methods are guided to move towards the preference of a decision maker. For instance, Abbass $[5,6]$ proposed an interactive evolutionary approach for MOPs with reference points. In the context of MOP, the task of GDM is to select the solutions most acceptable by the group among the nondominated set. We use group decision making for multi-objective problem (GDM-MOP) to indicate such a combination of group decision making and multi-objective problems.

The process of GDM-MOP is usually in the presence of uncertainty. On one hand, as the complexity of realworld decision problems and the limitation of DMs' background and expertise, it is difficult for a decision maker to precisely express his/her preference on each objective. On the other hand, the DMs' preferences might change over time according to various related factors, for example, the decision maker's attitude towards each objective or 
the changes on external environment. In such a case, the group solution may change because of the perturbation on decision maker's preference. However, in MOP, it is expected that the selected solution(s) can stay in the non-dominated set in the presence of any potential change. In other words, the solutions should be robust against the perturbations on decision maker's preference. Abbass and Bender [7] addressed such kind of problems where tradeoff between the objectives to be optimized varies over time and proposed the concept of Pareto Operating Curve. For similar problems, two robustness measures were suggested in Bui et al. [8].

Although the imprecise preference of decision maker can take many other forms, in this research, we focus on the GDM-MOP where the preferences are given by fuzzy numbers. The problem is referred to as fuzzy group decision making for multi-objective problem (FGDM-MOP). To the best of our knowledge, there is no other literature that addressed such kind of problems. From the previous discussion, we may claim that in FGDM-MOP, two issues need to be addressed: the first is how acceptable is a solution by the decision group? The second is how robust is a solution against possible changes or perturbations? In the proposed approach, we employ consensus and robustness to indicate the degrees of the two above mentioned issues. In many cases, the consensus and robustness degrees are in conflict with each other. Thus, the FGDM-MOP itself can be considered as a multi-objective problem. This paper presents the model and procedure of FGDM-MOP and suggests consensus and robustness measures. A multiobjective evolutionary approach is proposed to solve the problem.

The remaining sections of the paper are organized as follows. Section 2 briefly reviews the related work on group decision making and multi-objective problems. The mathematical model of FGDM-MOP is presented in Section 3. The measures of consensus and robustness are suggested in Section 4. Section 5 describes the evolutionary approach to solve FGDM-MOP. The experimental results with a modified benchmark function are reported in Section 6. Finally, conclusions and future works are discussed in Section 7.

\section{Related Work}

2.1. Multiobjective Optimization. Most real-world problems are characterized by multiple, noncommensurate, and often conflicting objectives [9]. The term multi-objective optimization problem (MOP) is used to refer to such kind of problem. In MOP, there are two or more conflicting objectives that needed to be optimized simultaneously. Formally, a MOP can be written as follows [4]:

$$
\begin{array}{ll}
\min & f(x)=\left(f_{1}(x), f_{2}(x), \ldots, f_{k}(x)\right) \\
\text { s.t. } & x \in X
\end{array}
$$

where $X \subset \mathbb{R}^{n}$ is a feasible set of decision variables and $f: \mathbb{R}^{n} \rightarrow \mathbb{R}^{k}$. The $n$-dimensional space $\mathbb{R}^{n}$ is called a variable space, and the functions $f_{i}(i=1, \ldots, k)$ are objective functions.
The solutions of MOP posed by (1) are called noninferior, non-dominated, efficient, or Pareto optimal solutions [4, 911]. This paper uses the naming convention of non-dominated or Pareto optimal solutions. A solution is called a Pareto optimal solution if there exists no other feasible solution which would decrease some criterion without causing a simultaneous increase in at least one other criterion [11]. To be more specific, we formally define the dominance and nondominated concepts as follows [4].

Definition 1. In (1), a vector $f(x), x \in X$, is said to dominate another vector, $f(y), y \in X$, if $f_{i}(x) \leq f_{i}(y)$ for all $i=$ $1, \ldots, k$, and the inequality is strict for at least one $i$.

Definition 2. In (1), a vector $f\left(x^{*}\right), x^{*} \in X$, is non-dominated if there exists no other $x \in X$ such that $f(x)$ dominates $f\left(x^{*}\right)$.

A set which consists of all non-dominated solutions is called a non-dominated set. The topology of the set of nondominated solutions in the objective space forms a curve that is known as the Pareto curve [7].

Evolutionary algorithms (EAs) have received considerable attention in dealing with MOPs during the last two decades as they are advantageous comparing with mathematical programming techniques. Currently, a wide variety of multi-objective evolutionary algorithms (MOEAs) are available in the literature. The representative MOEAs include SPEA [12], SPEA2 [13], PAES [14], and NSGA-II [15]. For a brief history of MOEAs, readers are referred to Coello [11].

From the definition of non-dominated solutions, one may notice that any choice among the set of non-dominated solutions could be difficult, unless we have additional information about the decision maker's preference structure [4]. Furthermore, in many cases, DMs might be interested in only some parts of the Pareto curve. Then, there is no need to visualize the whole Pareto curve, which is time intensive when the solution space is huge. Thus, it is important to utilize preference information during the optimization process. To perform this task, several preference-based multi-objective evolutionary algorithms are proposed and applied in recent years [4, 16-21]. However, most (if not all) existing literatures of preference-based multi-objective approaches neglect the following issues: (1) the preference is from a group of DMs, (2) the preference could be imprecise, and (3) the final solutions' ability to deal with any change on preference. This paper is aiming at simultaneously addressing the previous issues, which might be the first attempt in the field.

2.2. Group Decision Making. As its practical importance, group decision making has received great attention in many application areas, such as situation assessment [22], accident evaluation [23], emergency management [3], product development [24], and alternative selection [25]. The aim of group decision making is to find a group satisfactory solution which is most acceptable by the group individuals as a whole [26]. The procedure of GDM usually consists of two processes [27-31]: consensus process and selection process. The former is used to obtain the maximum degree of agreement between the set of DMs on the solution set of alternatives. Such 
a process is defined as a dynamic and iterative group discussion process, coordinated by a moderator helping DMs bring their opinions closer [31]. The latter refers to obtain the solution set of alternatives according to the collective opinions of DMs.

Since consensus degree is employed to identify the agreement level amongst all DMs, it is preferable that the set of DMs can achieve the maximum consensus before applying the selection process [31]. Thus, how to obtain the maximum consensus for the given problem is a hot issue in GDM, and various approaches and methods have been developed in recent years. Hsu and Chen [32] presented a procedure for aggregating the individual opinions into a group consensus opinion. Herrera et al. [28] presented a consensus model in group decision making under linguistic assessments. Herrera et al. [33] proposed a model for the consensus reaching problem in heterogeneous group decision making. The model contains two types of linguistic consensus measures: linguistic consensus degrees and linguistic proximities to guide the consensus reaching process. A similar work was reported in Herrera-Viedma et al. [34], where a model of consensus support system was presented to assist the DMs in all phases of the consensus reaching process of group decision making problems with multigranular linguistic preference relations. Mata et al. [35] proposed an adaptive consensus support system model for the group decision making problems defined in multigranular linguistic contexts. Xu [36] developed an automatic approach to reaching consensus among group opinions for multiple attribute group decision making problems. It is worthwhile to note that in group decision making problems, besides consensus degree, some other measures might be used to indicate the final solution(s) from different aspects. Wu and $\mathrm{Xu}$ [37] studied individual consistency and group consensus in the process of group decision making with multiplicative preference relations and provided a decision support model to aid the group consensus process while keeping an acceptable individual consistency for each decision maker. In the present paper, we simultaneously consider consensus and robustness degrees of a solution for FGDM-MOP, providing the tradeoff between the solutions' acceptable degree among DMs and the ability to cope with any change on preferences.

\section{Problem Formulation}

The result of multi-objective optimization problem, shown as in (1), is a set of non-dominated solutions, denoted $N D$. The task of multi-objective decision making is to select a final solution among the non-dominated set.

It is usual that the selection process involves multiple DMs. Suppose the decision group consists of $m$ DMs, denoted as $D_{j}(j=1,2, \ldots, m)$. The weight vector of DMs is represented as $\omega=\left(\omega_{1}, \omega_{2}, \ldots, \omega_{m}\right)$, satisfying $\sum_{j=1}^{m} \omega_{j}=1$. DMs express their preference by reference points in objective space, denoted as $R_{j}=\left(r_{j 1}, \ldots, r_{j k}\right)$, where $r_{j i}(i=1, \ldots, k)$ is the reference value of $i$ th objective function given by the $j$ th $\mathrm{DM}$. The reference values given by DMs for each objective can be represented as triangular fuzzy numbers [38]; each value can be expressed as a triple $r_{j i}=\left(r_{j i}^{\text {lower }}, r_{j i}^{\text {most }}, r_{j i}^{\text {upper }}\right)$ with its membership function defined as

$$
\mu_{A}(r)= \begin{cases}\frac{\left(r-r_{j i}^{\text {lower }}\right)}{\left(r_{j i}^{\text {most }}-r_{j i}^{\text {lower }}\right)}, & r_{j i}^{\text {lower }} \leq r \leq \mathrm{r}_{j i}^{\text {most }}, \\ \frac{\left(r_{j i}^{\text {upper }}-r\right)}{\left(r_{j i}^{\text {upper }}-r_{j i}^{\text {most }}\right)}, & r_{j i}^{\text {most }} \leq r \leq r_{j i}^{\text {upper }}, \\ 0, & \text { otherwise, }\end{cases}
$$

where $r_{j i}^{\text {most }}$ is the most possible value of the fuzzy number $A$ and $r_{j i}^{\text {lower }}$ and $r_{j i}^{\text {upper }}$ are, respectively, the lower and upper bounds, used to reflect the fuzziness of the DMs' preference.

Given the preferences of DMs, the consensus degree of each solution in non-dominated set is denoted as $C_{p}(p=$ $1, \ldots, N)$, where $N$ is the number of non-dominated solutions. For each solution in non-dominated set, its ability to deal with the changes on DMs' preference is termed as robustness and is denoted as $\operatorname{Rob}_{p}(p=1, \ldots, N)$.

Thus, in FGDM-MOP, we need to find solutions which are both acceptable by the group and robust against fuzzy preferences of DMs. The decision making problem can be formally modeled as follows:

$$
\begin{array}{ll}
\text { obj.: } & \max C(x) \\
& \max \operatorname{Rob}(x) .
\end{array}
$$

The calculation of consensus and robustness will be presented in the following section.

\section{Consensus and Robustness Measures}

Usually, in group decision making, consensus is employed to measure the closeness among DMs' opinions [31]. For each final selected solution, it is expected to be as close to the collective opinion as possible. The term of consensus employed in this paper is slightly different from other literatures. In Herrera et al. [28], Herrera-Viedma et al. [29] consensus degree is used to reflect the closeness of the DMs' preferences on a set of alternatives. While in our problem, the DMs' preferences are expressed as reference points in the objective space. Thus, we use consensus to reflect not only the closeness among DMs' preferences, but also the distance of each solution in the Pareto curve to the collective preference. Since the preferences of DMs are fuzzy, the robustness is employed to measure each solution's ability of dealing with any change on the preferences.

4.1. Consensus Measure. Once the reference points are given, the consensus degree for each solution is calculated as follows.

(1) Distance between the solution and each reference point.

For each solution $x$, the objective values are $\left(f_{1}(x)\right.$, $\left.\ldots, f_{k}(x)\right)$, where $k$ is the number of objectives. Since 
the reference value on each objective is expressed by fuzzy number, the relative distance between the solution and reference point on each objective is given by a fuzzy distance. For the reference value on the $i$ th objective given by decision maker $D_{j}$, that is, $r_{j i}=\left(r_{j i}^{\text {lower }}, r_{j i}^{\text {most }}, r_{j i}^{\text {upper }}\right)$, we employ the concept of vertex method [39] to calculate the distance, given as follows:

$$
d_{j i}(x)=\frac{\sqrt{(1 / 3)\left(f_{i}(x)-r_{j i}^{\text {lower }}\right)^{2}+(1 / 3)\left(f_{i}(x)-r_{j i}^{\text {most }}\right)^{2}+(1 / 3)\left(f_{i}(x)-r_{j i}^{\text {upper }}\right)^{2}}}{f_{i}(x)^{\max }-f_{i}(x)^{\min }}
$$

where $j=1, \ldots, m, i=1, \ldots, k, f_{i}(x)^{\max }$, and $f_{i}(x)^{\min }$, respectively, represent maximum and minimum values of the $i$ th objective function in a given set.

By aggregating the distance measures on all objectives, the distance between the solution and the reference point given by decision maker $D_{j}$ can be obtained and given as follows:

$$
d_{j}(x)=\phi\left(d_{j i}(x), i=1, \ldots, k\right),
$$

where $\phi$ is aggregation function, which is arithmetic mean in this research. However, different aggregation operators could be used. For example, if a weight vector is used in objective functions, the aggregation operator could be replaced with weighted aggregation.

(2) Aggregation of distances to obtain the consensus degree.

The distance $d_{j}(x)$ indicates how far the solution is from the individual decision maker's preference. In order to obtain the consensus measure among the group, the distances from all DMs' reference points need to be considered. In our case, we use additive weight aggregation operator to calculate the consensus degree, given as follows:

$$
\mathrm{cd}(x)=\sum_{j=1}^{m} \omega_{j} d_{j}(x)
$$

where $m$ is the number of decision makers.

4.2. Robustness Measure. In order to evaluate the effect of perturbations in the objective space of MOP, Bui et al. [8] defined the preference robustness as follows: preference robustness of a non-dominated solution is defined as the minimum transition costs in decision space when the solution is perturbed in objective space. Preference robustness is specifically tailored for MOP and indicates the closeness of other non-dominated solutions in the decision space.

Following the principle of Bui et al. [8], the term of robustness used in this paper refers to the preference robustness. Clearly, for each solution, given a neighborhood radius $\delta$ in the $n$-dimensional decision space, if there are more neighbors and smaller distances, the solution is expected to be more capable of moving to another non-dominated solution with a less transition cost. It is worthwhile to note that the transition cost varies depending on problem domain [8]. Thus, we modify the preference robustness measure and approximate it as follows:

$$
\operatorname{rd}(x)=\frac{1}{\left|N D_{\delta}\right|+\epsilon}+\frac{\sum_{y \in N D_{\delta}} d(x, y)}{\left|N D_{\delta}\right|+\epsilon},
$$

where $N D_{\delta}$ represents the set of all non-dominated solutions in the area of neighborhood with radius $\delta$ of the nondominated solution $x,\left|N D_{\delta}\right|$ indicates the number of nondominated solutions in the neighborhood, and $\epsilon$ is a small number used to avoid potential singularities in the denominator, which is set to $1 E-06 . d(x, y)$ is the relative distance between the solution $x$ and its neighbor $y$, given as follows:

$$
d(x, y)=\frac{\sum_{q=1}^{n}\left(\left|x_{q}-y_{q}\right| /\left(x_{q}^{\max }-x_{q}^{\min }\right)\right)}{n}
$$

where $x_{q}^{\max }$ and $x_{q}^{\min }$, respectively, indicate the maximum and minimum values of the qth decision variable in the nondominated set. It can be seen from (7) that the calculation of robustness $\operatorname{rd}(x)$ consists of two parts: the first part reflects the number of neighbors in the neighborhood, while the second part indicates the average distance of the nondominated solution from its neighbors.

\section{Evolutionary Approach for GDM-MOO}

It is clear that for both consensus and robustness measures, the smaller $\operatorname{cd}(x)$ and $\operatorname{rd}(x)$, the better the consensus and robustness a solution has. Thus, the decision making problem presented in (3) can be replaced as follows:

$$
\begin{array}{ll}
\text { obj.: } & \min c d(x) \\
& \min \operatorname{rd}(x) .
\end{array}
$$

We employ one of classical multi-objective evolutionary algorithms, NSGA-II [15], as the optimizer for multiobjective optimization problem. NSGA-II is an elitism-based multiobjective evolutionary algorithm, whose main feature is an elitism-preserving operation. In NSGA-II, the parent population and offspring are combined and sorted in order to generate a population for the next generation. A nondominated sorting mechanism is performed to classify the combined population into different ranks of nondomination. A crowding-distance assignment is employed to ensure that diversity is maintained among non-dominated solutions. 
TABLE 1: The fuzzy reference points given by DMs for M-BINH.

\begin{tabular}{lcc}
\hline Decision maker $\left(D_{j}\right)$ & $r_{j 1}$ & $r_{j 2}$ \\
\hline$D_{1}$ & $(36.0,40.0,44.6)$ & $(8.2,10.0,13.3)$ \\
$D_{2}$ & $(26.9,30.0,32.1)$ & $(13.0,15.0,19.5)$ \\
$D_{3}$ & $(16.6,20.0,25.6)$ & $(3.9,5.0,8.5)$ \\
$D_{4}$ & $(38.1,45.0,48.8)$ & $(12.4,15.0,17.3)$ \\
$D_{5}$ & $(18.3,20.0,21.1)$ & $(17.8,20.0,22.4)$ \\
\hline
\end{tabular}

In the original NSGA-II, the mechanism of dominance ranking is used to classify the population into a number of layers, such that the first layer is the non-dominated set in the population and with the rank value of 1 . Similarly, in the second layer, the solutions are non-dominated in the population with the first layer removed and with the rank value of 2 . The sorting procedure is continued until all solutions in the population are classified into a layer and assigned a rank value. In order to perform the decision making task and incorporate the DMs' preference information into the optimization process, we slightly modify this ranking procedure of NSGA-II.

The final solutions of problem in (9) is a subset of the solutions of original multi-objective optimization problem in (3). In other words, some of the solutions with the first nondominated rank will be excluded because they are dominated for (9). Thus, after each generation, we modify the nondominated rank of the obtained solutions as follows:

$$
\operatorname{Ind}_{p}^{\mathrm{rank}}= \begin{cases}\operatorname{Ind}_{p}^{\mathrm{rank}}, & \text { if } \operatorname{Ind}_{p}^{\mathrm{rank}}=1 \wedge \operatorname{Ind}_{p} \\ \operatorname{Ind} d_{p}^{\mathrm{rank}}+1, & \text { is nondominated for }\end{cases}
$$

where $\operatorname{Ind}_{p}$ is the $p$ th individual solution in the population and $\operatorname{Ind}_{p}^{\text {rank }}$ indicates the non-dominated rank of the solution Ind $_{p}$.

\section{Experimental Results}

We illustrate the proposed approach using and modifying a well-known benchmark function. We investigated the problem introduced by Binh and Korn [40] with two objectives, denoted as BINH and given as follows:

$$
\begin{aligned}
& \min f_{1}\left(x_{1}, x_{2}\right)=x_{1}^{2}+x_{2}^{2}, \\
& \min f_{2}\left(x_{1}, x_{2}\right)=\left(x_{1}-5\right)^{2}+\left(x_{2}-5\right)^{2},
\end{aligned}
$$

where $-5 \leq x_{1}, x_{2} \leq 10$.

In BINH, the solutions evenly spread in both decision and objective spaces. In order to illustrate the proposed approach clearer, we modified the original BINH as a many-to-one problem. Similar with in Bui et al. [8], the function's domain is divided into different parts. Each part uses a different resolution for forming intervals that are mapped to a single point in objective space. The modified BINH is denoted as $\mathrm{M}-\mathrm{BINH}$ and given as follows:

$$
\begin{gathered}
x_{p}^{c}=\frac{x_{p}^{\max }+x_{p}^{\min }}{2}, \\
r_{1-p}=0.2, \\
r_{2-p}=\frac{x_{p}}{x_{p}^{\max },} \\
x_{p}=\left\{\begin{array}{cc}
x_{p}, & \text { if } x_{p} \leq 1 \\
x_{p}^{\min }+\text { floor }\left(\frac{x_{p}-x_{p}^{\min }}{r_{1-p}}\right) r_{1-p}, & \text { if } x_{p}<x_{p}^{c} \\
x_{p}^{c}+\text { floor }\left(\frac{x_{p}-x_{p}^{c}}{r_{2-p}}\right) r_{2-p}, & \text { else, } \\
p=1,2, & \\
\min f_{1}\left(x_{1}, x_{2}\right)=x_{1}^{2}+x_{2}^{2}, & \\
\min f_{2}\left(x_{1}, x_{2}\right)=\left(x_{1}-5\right)^{2}+\left(x_{2}-5\right)^{2},
\end{array}\right.
\end{gathered}
$$

where $0 \leq x_{1}, x_{2} \leq 5, x_{p}^{\min }$ and $x_{p}^{\max }(p=1,2)$, respectively, indicate the lower and upper bounds of $x$-domain. $r_{1-p}$ and $r_{2-p}$ are resolutions for the different parts of the domain of $x_{p}$.

It is supposed that there are five DMs, whose weight coefficients are $(0.12,0.20,0.18,0.22,0.28)$. The fuzzy reference points given by each decision maker are presented in Table 1 .

6.1. Parameter Setting. The population size was set to 100 , the crossover rate was 0.95 , and the mutation rate was $1 / n$, where $n$ is the number of decision variables. The distribution indices for crossover and mutation operators were, respectively, set to $\eta_{c}=20$ and $\eta_{m}=10$. The evolution process was terminated after 400 generations. In the calculation of robustness, the radius of neighborhood $\delta$ was set to 0.08 . Furthermore, in order to alleviate the effect of algorithm's stochastic nature, each experiment is run repeatedly for 10 times with different random seeds.

6.2. Results Analysis. At first, we report the obtained nondominated solutions of M-BINH in both decision and objective spaces, respectively, presented in Figures 1 and 2. From the figures, one can see that the non-dominated solutions are discontinuous and each part with different resolution. In particular, in the domain $x_{1}, x_{2} \in[0,1]$, the non-dominated solutions are dense, continuous, and evenly spread in both decision and objective spaces. While for $x_{1}, x_{2} \in(1,5]$, the non-dominated solutions are divided into several parts with different densities.

With reference points, we can obtain the non-dominated solutions of interest to DMs, shown as in Figure 3. In the figure, the most preferable values of the fuzzy reference points given by DMs are represented as black stars, while the nondominated solutions obtained with preferences are indicated by blue triangles. It can be seen that with DMs' preference, the 


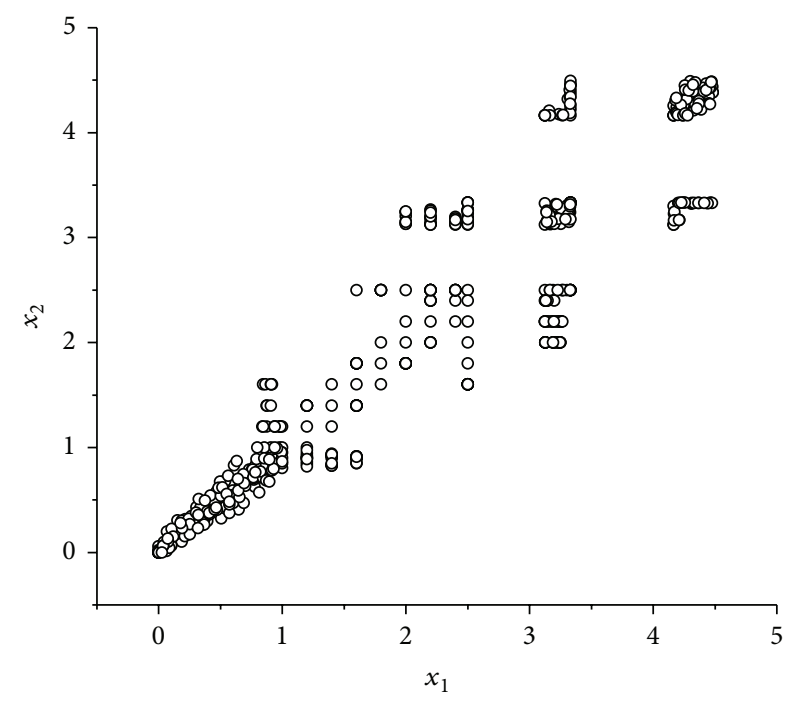

FIGURE 1: The obtained nondominated solutions of M-BINH problem, projected in decision space.

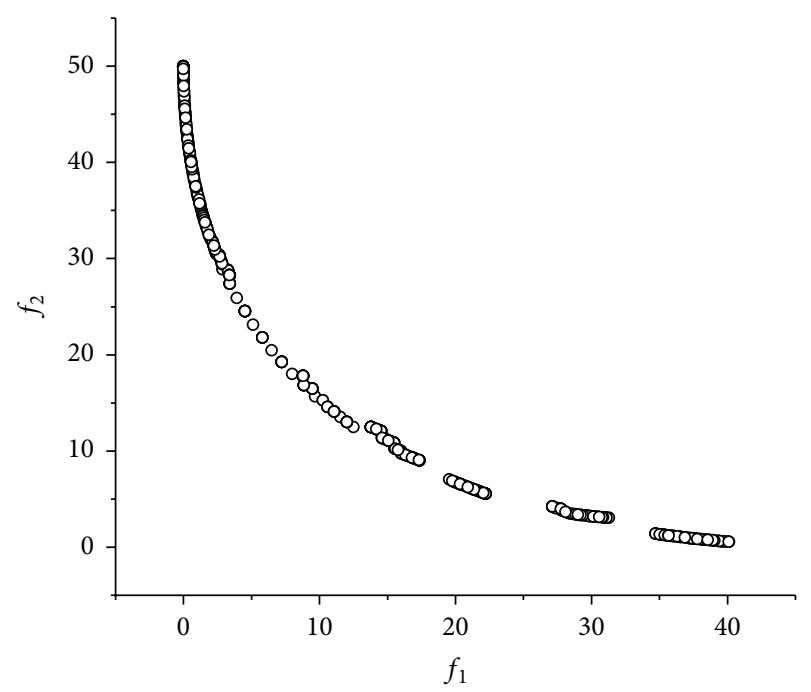

FIGURE 2: The obtained non-dominated solutions of M-BINH problem, projected in objective space.

obtained solutions are a subset of the non-dominated solutions obtained without any preference. One may notice that some solutions far from the reference points are included, for example, the solutions with lower values on objective $f_{1}$ and higher values on $f_{2}$. This is because these solutions provide higher values on robustness measures. Since the robustness of a solution is measured in decision space, we also depicted the projection of the non-dominated solutions obtained with reference points on decision space, shown as in Figure 4. Similarly, the solutions obtained with reference points are denoted as blue triangles. From Figure 4, one can see that the final selected non-dominated solutions are the ones which are surrounded by other non-dominated solutions. Thus, these non-dominated solutions can easily move to another position

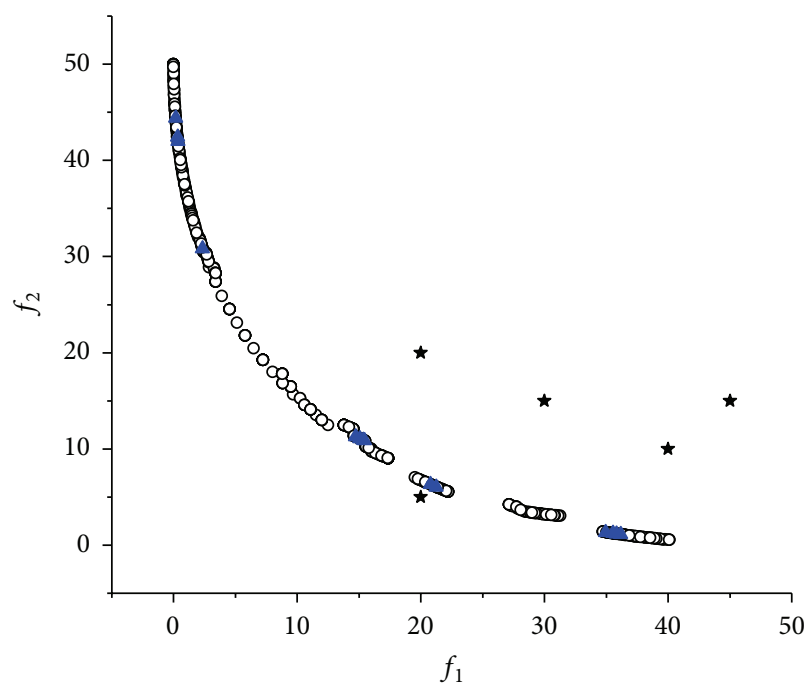

Figure 3: The obtained non-dominated solutions of M-BINH problem with reference points, projected in objective space.

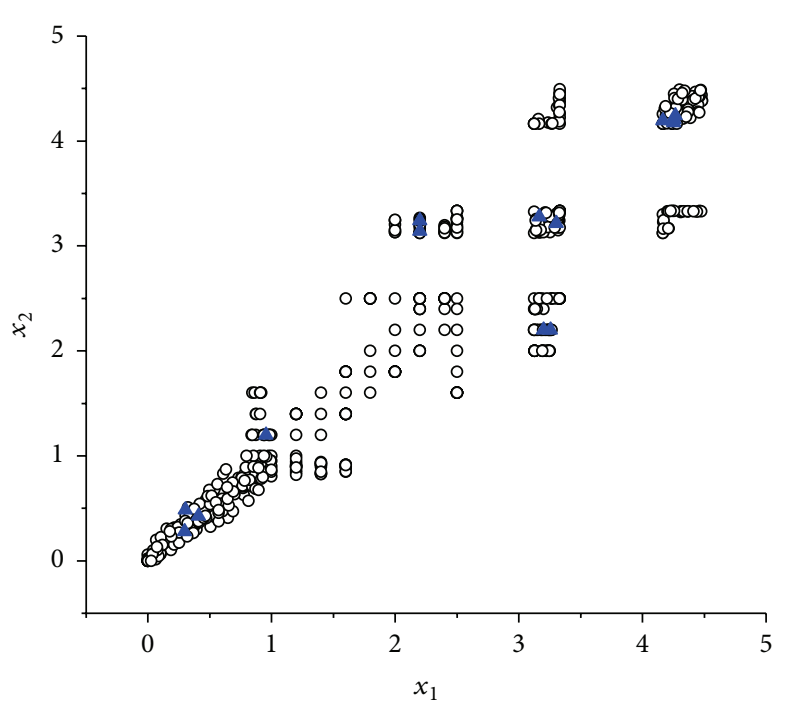

FIGURE 4: The obtained non-dominated solutions of M-BINH problem with reference points, projected in decision space.

in the non-dominated set in the presence of perturbation on decision space or DMs' preference.

As indicated earlier, for each solution, consensus and robustness measures are in conflict with each other to a certain degree. In other words, in the final selected non-dominated set, the values of consensus and robustness of all solutions form another non-dominated set in consensus-robustness space. Figure 5 shows such a confliction between consensus and robustness. In order to clearly present the mapping among decision space, objective space, and consensus-robustness space, Table 2 lists the decision variables, objective functions, and consensus and robustness measures of the final selected non-dominated set. 
TABLE 2: The decision variables, objective functions, and consensus and robustness measures of the final selected nondominated solutions for M-BINH.

\begin{tabular}{|c|c|c|c|c|c|c|}
\hline Index & $x_{1}$ & $x_{2}$ & $f_{1}$ & $f_{2}$ & $\mathrm{~cd}$ & $\mathrm{rd}$ \\
\hline 1 & 3.2576 & 2.2000 & 15.4516 & 10.8761 & 0.2412 & 0.1538 \\
\hline 2 & 3.2006 & 2.2000 & 15.0836 & 11.0780 & 0.2449 & 0.1511 \\
\hline 3 & 3.1648 & 3.2792 & 20.7687 & 6.3294 & 0.2112 & 0.1577 \\
\hline 4 & 0.3035 & 0.4851 & 0.3274 & 42.4414 & 0.6620 & 0.1122 \\
\hline 5 & 0.2967 & 0.2782 & 0.1654 & 44.4161 & 0.6840 & 0.1069 \\
\hline 6 & 2.2000 & 3.2415 & 15.3476 & 10.9322 & 0.2399 & 0.1539 \\
\hline 7 & 2.2000 & 3.1431 & 14.7190 & 11.2881 & 0.2461 & 0.1498 \\
\hline 8 & 4.2535 & 4.1775 & 35.5441 & 1.2337 & 0.2687 & 0.1329 \\
\hline 9 & 4.1674 & 4.1938 & 34.9552 & 1.3432 & 0.2652 & 0.1484 \\
\hline 10 & 4.2658 & 4.2414 & 36.1870 & 1.1144 & 0.2726 & 0.1146 \\
\hline 11 & 4.2581 & 4.2069 & 35.8298 & 1.1794 & 0.2704 & 0.1225 \\
\hline 12 & 0.9549 & 1.2000 & 2.35185 & 30.8028 & 0.5242 & 0.1133 \\
\hline 13 & 0.4089 & 0.4281 & 0.3505 & 41.9797 & 0.6487 & 0.1125 \\
\hline 14 & 3.3027 & 3.2169 & 21.2564 & 6.0602 & 0.2105 & 0.2155 \\
\hline
\end{tabular}

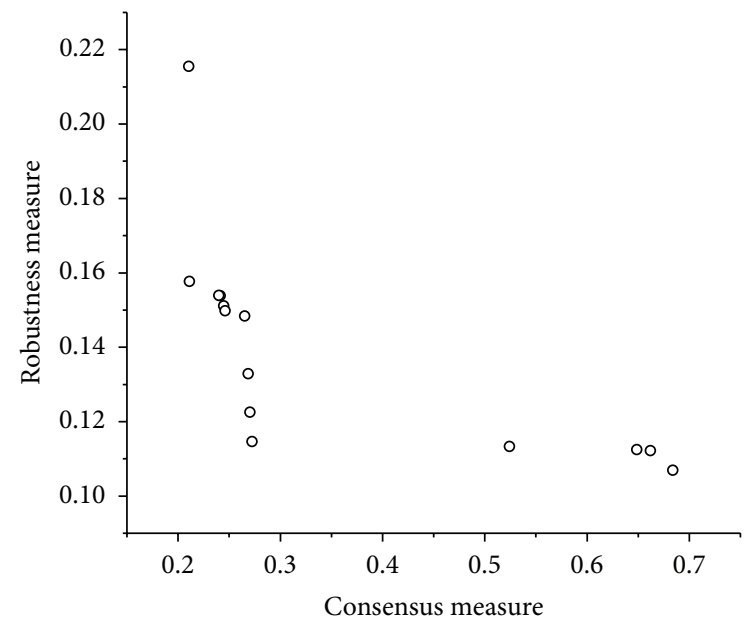

FIGURE 5: The consensus and robustness measures of the obtained non-dominated solutions of M-BINH problem with reference points.

\section{Conclusion}

In many real-world decision problems, a set of decision makers need to trade off between multiple conflicting objectives then provide their preferences to select the final solution(s). This paper studies such an amalgamation of group decision making (GDM) and multi-objective problems (MOP), referred to as GDM-MOP. As the limitation of the DMs' background and/or knowledge structure, it is usually hard for them to give precise preference information. Thus, the GDM-MOP with fuzzy preference is addressed in this research, called as fuzzy GDM-MOP (FGDM-MOP). In the context of GDM, consensus is used to indicate the distance of a solution from the group preference. In the presence of uncertain preference, robustness is suggested to measure the ability of coping with change on preference. Thus, in the process of FGDM-MOP, it is preferable to pursue both great consensus and robustness degrees. This paper firstly describes the mathematical model of FGDM-MOP. Then, a new multi-objective model is introduced for FGDM-MOP, where the measures of consensus and robustness are simultaneously considered. To obtain the refined non-dominated set of new established multi-objective model, one of the classical multi-objective evolutionary algorithms-NSGAII-is employed and modified to solve the problem. However, how to identify the final solution with the consideration of consensus and robustness highly depends on the decision makers' preferences on different measures. We leave this new decision making problem to our future research. We examine a benchmark function to illustrate the concept and procedure of the proposed approach. In our future study, some real cases will be addressed through the proposed approach.

\section{Acknowledgment}

This research was supported in part by the National Natural Science Foundation of China under the Contract no. 71101096 and 71001104 .

\section{References}

[1] U. Bose, A. M. Davey, and D. L. Olson, "Multi-attribute utility methods in group decision making: past applications and potential for inclusion in GDSS," Omega, vol. 25, no. 6, pp. 691706, 1997.

[2] J. Lu, G. Zhang, D. Ruan, and F. Wu, Multi-Objective Group Decision Making: Methods, Software and Applications with Fuzzy Set Technology, Imperial College Press, London, UK, 2007.

[3] G. Zhang, J. Ma, and J. Lu, "Emergency management evaluation by a fuzzy multi-criteria group decision support system," Stochastic Environmental Research and Risk Assessment, vol. 23, no. 4, pp. 517-527, 2009.

[4] L. Thiele, K. Miettinen, P. J. Korhonen, and J. Molina, "A preference-based evolutionary algorithm for multi-objective 
optimization," Evolutionary Computation, vol. 17, no. 3, pp. 411436, 2009.

[5] H. A. Abbass, "An inexpensive cognitive approach for biobjective optimization using bliss points and interaction," in Proceedings of the 8th International Conference on Parallel Problem Solving From Nature (PPSN), vol. 3242 of Lecture Notes in Computer Science, pp. 712-721, Springer, New York, NY, USA, 2004.

[6] H. A. Abbass, "An economical cognitive approach for biobjective optimization using bliss points, visualization, and interaction," Soft Computing, vol. 10, no. 8, pp. 687-698, 2006.

[7] H. A. Abbass and A. Bender, "The Pareto operating curve for risk minimization in life and robotics," Artificial Life Robotics, vol. 14, no. 4, pp. 449-452, 2009.

[8] L. T. Bui, H. A. Abbass, M. Barlow, and A. Bender, "Robustness against the decision-maker's attitude to risk in problems with conflicting objectives," IEEE Transactions on Evolutionary Computation, vol. 16, no. 1, pp. 1-19, 2012.

[9] Y. Y. Haimes, Risk Modeling, Assessment, and Management, John Wiley \& Sons, Hoboken, NJ, USA, 3rd edition, 2008.

[10] M. Ehrgott and X. Gandibleux, "Approximative solution methods for multiobjective combinatorial optimization," Top, vol. 12, no. 1, pp. 1-63, 2004.

[11] C. A. C. Coello, "Evolutionary multi-objective optimization: a historical view of the field," IEEE Computational Intelligence Magazine, vol. 1, no. 1, pp. 28-36, 2006.

[12] E. Zitzler and L. Thiele, "Multiobjective evolutionary algorithms: a comparative case study and the strength Pareto approach," IEEE Transactions on Evolutionary Computation, vol. 3, no. 4, pp. 257-271, 1999.

[13] E. Zitzler, M. Laumanns, and L. Thiele, "Spea2: Improving the strenth Pareto evolutionary algorithm," in Evolutionary Methods for Design, Optimization and Control with Applications to Industrial Problems (EUROGEN 2001), K. Giannakoglou, Ed., pp. 95-100, International Center for Numerical Methods in Engineering, Athens, Greece, 2002.

[14] J. D. Knowles and D. W. Corne, "Approximating the nondominated front using the Pareto archived evolution strategy," Evolutionary Computation, vol. 8, no. 2, pp. 149-172, 2000.

[15] K. Deb, A. Pratap, S. Agarwal, and T. Meyarivan, "A fast and elitist multiobjective genetic algorithm: NSGA-II," IEEE Transactions on Evolutionary Computation, vol. 6, no. 2, pp. 182197, 2002.

[16] S. Phelps and M. Köksalan, "An interactive evolutionary metaheuristic for multiobjective combinatorial optimization," Management Science, vol. 49, no. 12, pp. 1726-1738, 2003.

[17] K. Deb and J. Sundar, "Reference point based multi-objective optimization using evolutionary algorithms," in Proceedings of the 8th Annual Genetic and Evolutionary Computation Conference (GECCO '06), pp. 635-642, Seattle, Wash, USA, July 2006.

[18] K. Deb and S. Chaudhuri, "I-MODE: an interactive multiobjective optimization and decision-making using evolutionary methods," in Evolutionary Multi-Criterion Optimization, S. Obayashi, Ed., vol. 4403 of Lecture Notes in Computer Science, pp. 788-802, Springer, Berlin, Germany, 2007.

[19] J. Hakanen, Y. Kawajiri, K. Miettinen, and L. T. Biegler, "Interactive multi-objective optimization for simulated moving bed processes," Control and Cybernetics, vol. 36, no. 2, pp. 283-302, 2007.

[20] S. Chaudhuri and K. Deb, "An interactive evolutionary multi-objective optimization and decision making procedure," Applied Soft Computing Journal, vol. 10, no. 2, pp. 496-511, 2010.
[21] J. Xiong, K. W. Yang, J. Liu, Q. S. Zhao, and Y. W. Chen, "A twostage preference-based evolutionary multi-objective approach for capability planning problems," Knowledge-Based Systems, vol. 31, pp. 128-139, 2012.

[22] J. Lu, G. Zhang, and D. Ruan, "Intelligent multi-criteria fuzzy group decision-making for situation assessments," Soft Computing, vol. 12, no. 3, pp. 289-299, 2008.

[23] R. A. Krohling and V. C. Campanharo, "Fuzzy TOPSIS for group decision making: a case study for accidents with oil spill in the sea," Expert Systems with Applications, vol. 38, no. 4, pp. 41904197, 2011.

[24] J. Lu, J. Ma, G. Zhang, Y. Zhu, X. Zeng, and L. Koehl, "Theme-based comprehensive evaluation in new product development using fuzzy hierarchical criteria group decision-making method," IEEE Transactions on Industrial Electronics, vol. 58, no. 6, pp. 2236-2246, 2011.

[25] J. Xiong, Y. Chen, K. Yang, and J. Liu, "A decision support model for multi-attribute group decision making using a multi-objective optimization approach," International Journal of Computational Intelligence Systems, vol. 6, no. 2, pp. 337-353, 2013.

[26] J. Ma, J. Lu, and G. Zhang, "Decider: a fuzzy multi-criteria group decision support system," Knowledge-Based Systems, vol. 23, no. 1, pp. 23-31, 2010.

[27] J. Kacprzyk, M. Fedrizzi, and H. Nurmi, "Group decision making and consensus under fuzzy preferences and fuzzy majority," Fuzzy Sets and Systems, vol. 49, no. 1, pp. 21-31, 1992.

[28] F. Herrera, E. Herrera-Viedma, and J. L. Verdegay, "A model of consensus in group decision making under linguistic assessments," Fuzzy Sets and Systems, vol. 78, no. 1, pp. 73-87, 1996.

[29] E. Herrera-Viedma, F. Herrera, and F. Chiclana, "A consensus model for multiperson decision making with different preference structures," IEEE Transactions on Systems, Man, and Cybernetics A, vol. 32, no. 3, pp. 394-402, 2002.

[30] S. Alonso, E. Herrera-Viedma, F. Chiclana, and F. Herrera, "A web based consensus support system for group decision making problems and incomplete preferences," Information Sciences, vol. 180, no. 23, pp. 4477-4495, 2010.

[31] F. J. Cabrerizo, J. M. Moreno, I. J. Pérez, and E. Herrera-Viedma, "Analyzing consensus approaches in fuzzy group decision making: advantages and drawbacks," Soft Computing, vol. 14, no. 5, pp. 451-463, 2010.

[32] H. M. Hsu and C. T. Chen, "Aggregation of fuzzy opinions under group decision making," Fuzzy Sets and Systems, vol. 79, no. 3, pp. 279-285, 1996.

[33] F. Herrera, E. Herrera-Viedma, and J. L. Verdegay, "Linguistic measures based on fuzzy coincidence for reaching consensus in group decision making," International Journal of Approximate Reasoning, vol. 16, no. 3-4, pp. 309-334, 1997.

[34] E. Herrera-Viedma, L. Martínez, F. Mata, and F. Chiclana, "A consensus support system model for group decision-making problems with multigranular linguistic preference relations," IEEE Transactions on Fuzzy Systems, vol. 13, no. 5, pp. 644-658, 2005.

[35] F. Mata, L. Martínez, and E. Herrera-Viedma, "An adaptive consensus support model for group decision-making problems in a multigranular fuzzy linguistic context," IEEE Transactions on Fuzzy Systems, vol. 17, no. 2, pp. 279-290, 2009.

[36] Z. Xu, "An automatic approach to reaching consensus in multiple attribute group decision making," Computers and Industrial Engineering, vol. 56, no. 4, pp. 1369-1374, 2009. 
[37] Z. Wu and J. Xu, "A consistency and consensus based decision support model for group decision making with multiplicative preference relations," Decision Support Systems, vol. 52, no. 3, pp. 757-767, 2012.

[38] L. A. Zadeh, "Fuzzy sets," Information and Control, vol. 8, no. 3, pp. 338-353, 1965.

[39] C. T. Chen, "Extensions of the TOPSIS for group decisionmaking under fuzzy environment," Fuzzy Sets and Systems, vol. 114, no. 1, pp. 1-9, 2000.

[40] T. T. Binh and U. Korn, "An evolution strategy for the multiobjective optimization," in Proceedings of the 2nd International Conference on Genetic Algorithms, pp. 23-28, 1996. 


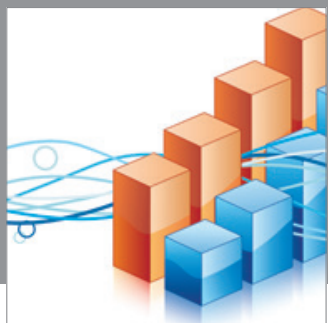

Advances in

Operations Research

mansans

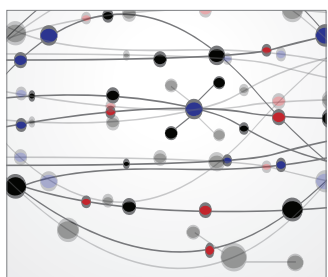

The Scientific World Journal
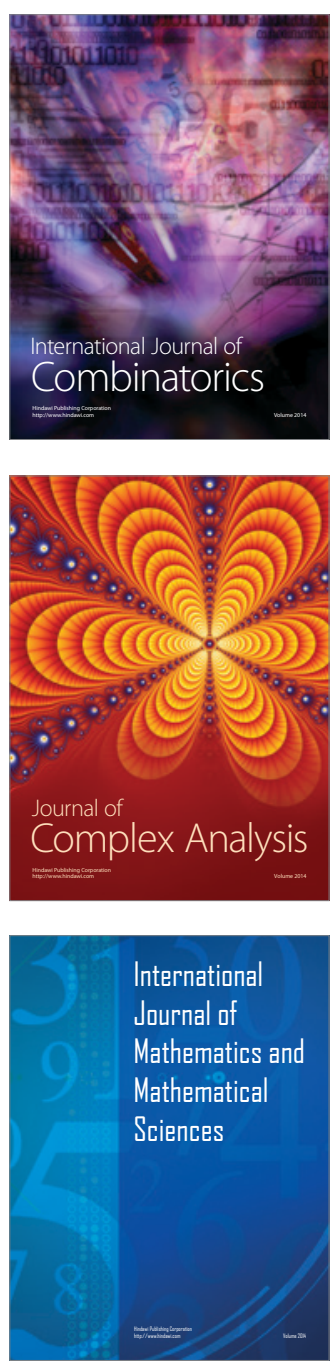
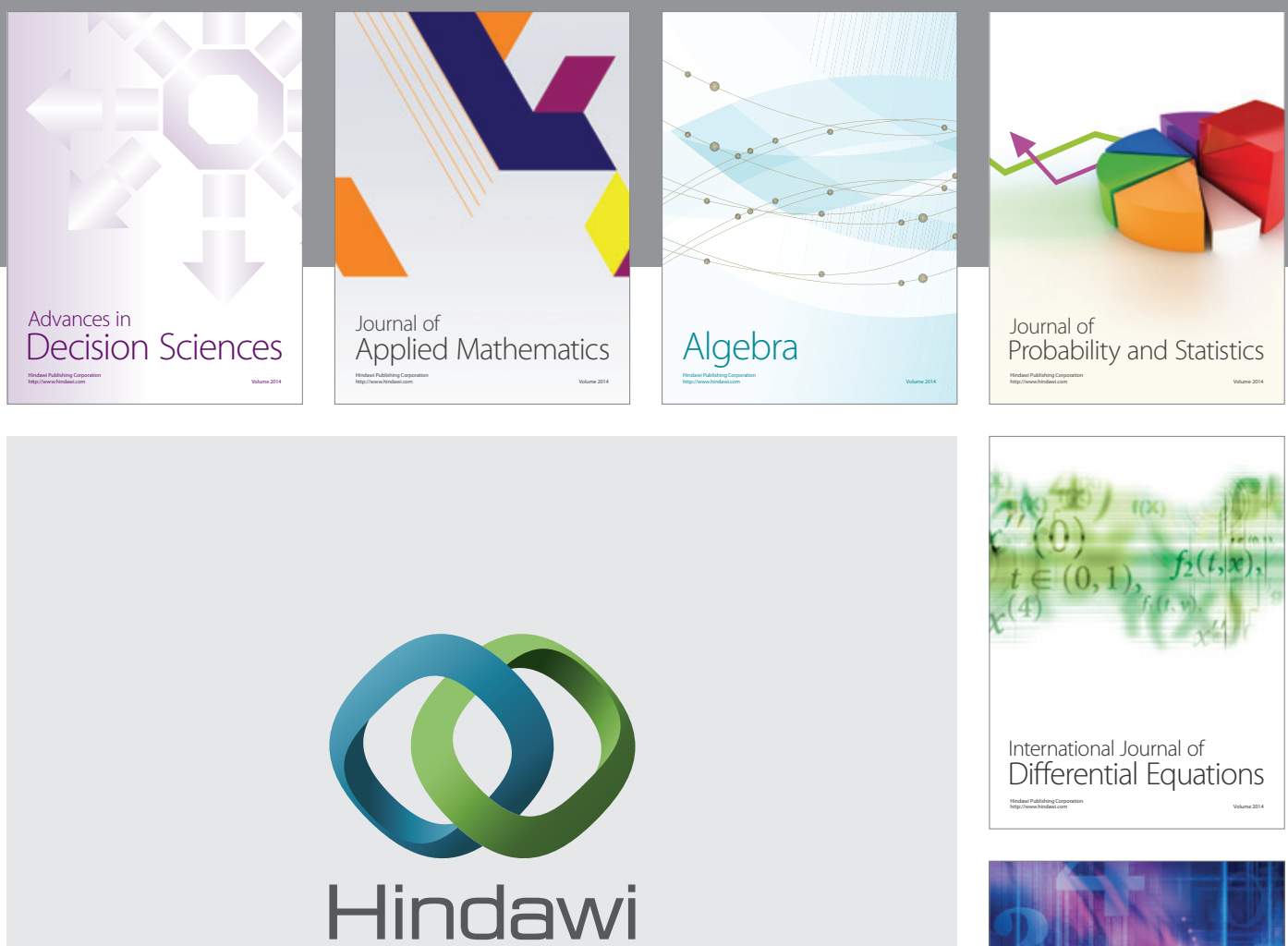

Submit your manuscripts at http://www.hindawi.com
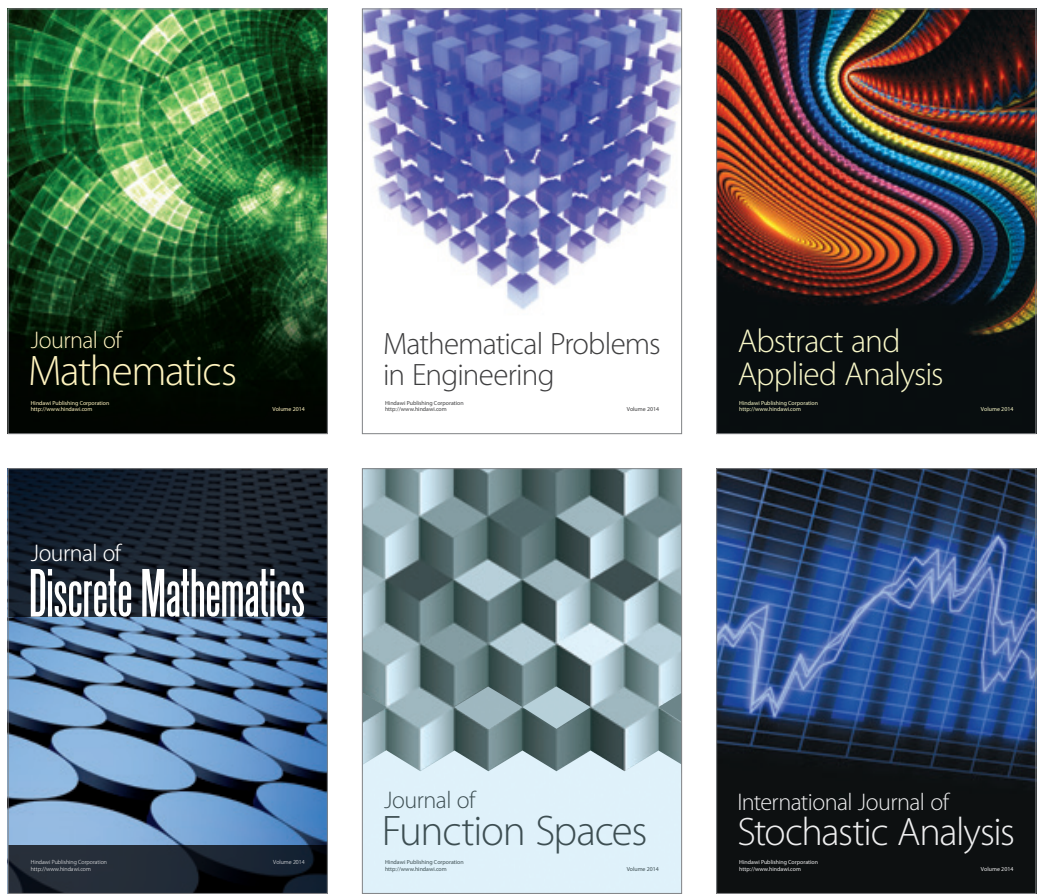

Journal of

Function Spaces

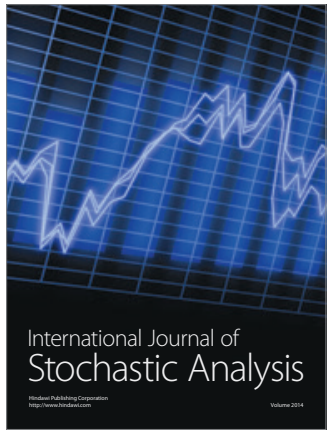

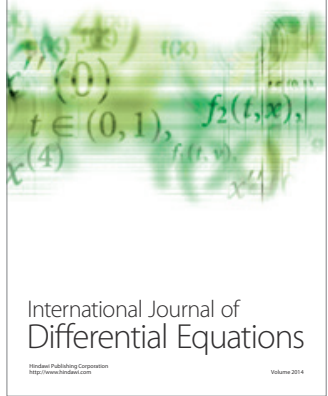
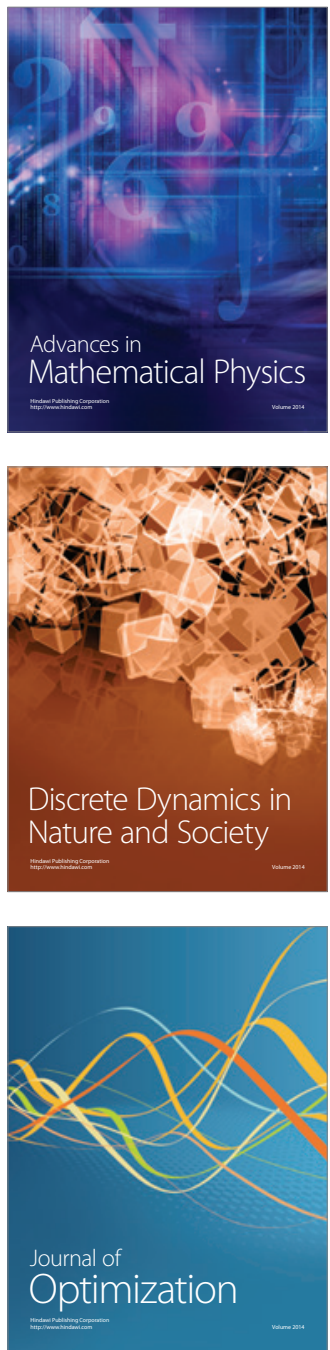